\title{
Ablación por radiofrecuencia en pediatría: características clínicas, electrofisiológicas y terapéuticas en un hospital pediátrico peruano
}

\author{
Luis Alfredo Melgar Quicaño(1) 1,a, ${ }^{*}$, Fredy Chipa Ccasani (iD,
}

Recibido: 01 de noviembre de 2021 Aceptado: 13 de diciembre de 2021

Filiación de los autores Instituto Nacional de Salud de Niño, San Borja. Lima, Perú. Médico cirujano, cardiólogo pediatra.

Médico cirujano, cardiólogo electrofisiólogo.

*Correspondencia

Luis Alfredo Melgar Quicaño

Av. Agustin de la Rosa Toro 1399

San Borja. Lima, Perú.

998648122

Correo

Imelgar@insnsb.gob.pe

luistrilenium@hotmail.com

Financiamiento

Autofinanciado.

Conflictos de interés

Los autores declaran no tener

conflictos de interés.

\section{Citar como:}

Melgar Quicaño LA, Chipa Ccasan F. Ablación por radiofrecuencia en pediatría: características clínicas, electrofisiológicas y terapéuticas en un hospital pediátrico peruan Arch Peru Cardiol Cir Cardiovasc. 2021;2(4):219-226. doi: 10.47487/ apcyccv.v2i4.167.

\section{RESUMEN}

Objetivo. Describir las características clínicas, electrofisiológicas y terapéuticas en una población pediátrica con taquicardia supraventricular, sometida a ablación por radiofrecuencia. Materiales y métodos. Estudio observacional, descriptivo, retrospectivo, de niños admitidos para el tratamiento de taquicardia supraventricular en el Instituto Nacional de Salud del Niño durante 2018 a 2021. Resultados. Se recolectaron datos de 62 procedimientos, que correspondían a 59 pacientes (media de edad: 9,91 años, 61\% varones), el $23 \%$ de procedimientos se realizaron en pacientes menores de $15 \mathrm{~kg}$, y dos fueron en menores de $5 \mathrm{~kg}$. El $63 \%$ de pacientes tenían un corazón estructuralmente normal, mientras que el $37 \%$ presentaban algún tipo de cardiopatía congénita, la más frecuente fue la anomalía de Ebstein. El grupo de medicamentos más usado fueron los betabloqueadores, principalmente el propranolol. Entre las arritmias tratadas, 21,7\% tuvieron síndrome de preexcitación, $33,3 \%$ portaban al menos una vía accesoria oculta, $5 \%$ correspondían a taquicardia reciprocante permanente de la unión, $5 \%$ a taquicardia intranodal, $11,7 \%$ taquicardia atrial, $10 \%$ flutter atrial, $5 \%$ tuvieron otros tipos de taquicardia y en cinco casos no se indujo taquicardia. De la totalidad de procedimientos, $76 \%$ correspondían a estudio electrofisiológico y ablación, cuatro pacientes recurrieron. Conclusiones. El sustrato de taquicardia supraventricular más frecuente en pacientes pediátricos fue la presencia de alguna vía accesoria. La ablación con catéter de radiofrecuencia pudo ser aplicado en estos pacientes con una alta tasa de éxito y una baja tasa de complicaciones.

Palabras clave: Taquicardia supraventricular; Ablación por catéter; Pediatría (fuente: DeCS BIREME)

\section{ABSTRACT}

Radiofrequency catheter ablation in pediatric population: clinical, electrophysiological and therapeutic characteristics in a Peruvian pediatric hospital

Objective. This study aims to describe the clinical, electrophysiological and therapeutic characteristics of pediatric patients with supraventricular tachycardia undergoing radiofrequency ablation. Materials and methods. Observational, descriptive, retrospective study of children admitted for supraventricular tachycardia treatment at the Instituto Nacional de Salud del Niño during 2018 to 2021. Results. Data from 62 procedures were collected, corresponding to 59 patients (mean age: 9.91 years, $61 \%$ male), $23 \%$ of procedures were performed in patients under $15 \mathrm{~kg}$, and two were in patients under $5 \mathrm{~kg} .63 \%$ of patients had a structurally normal heart, while $37 \%$ had some type of congenital heart disease, the most common being Ebstein's anomaly. The most widely used group of drugs were beta-blockers, mainly propranolol. Among the arrhythmias treated, $21.7 \%$ had preexcitation Syndrome, $33.3 \%$ had at least one hidden accessory pathway, $5 \%$ corresponded to permanent reciprocating junctional tachycardia, 5\% intranodal tachycardia, $11.7 \%$ atrial tachycardia, $10 \%$ atrial flutter, $5 \%$ had other types of tachycardia and in 5 cases no tachycardia was induced. Of the total procedures, $76 \%$ corresponded to electrophysiological study and ablation, four patients recurred. Conclusions. The most common supraventricular tachycardia substrate in children was the presence of some accessory pathway. Radiofrequency catheter ablation could be applied in children with a high success rate and a low complication rate.

Keywords: Tachycardia, supraventricular; Catheter ablation; Pediatrics (source: MeSH NLM). 


\section{Introducción}

Los trastornos del ritmo ocupan una parte importanteen la atención de cardiología pediátrica $y$, muchas veces, son susceptibles de un tratamiento curativo definitivo mediante técnicas invasivas ${ }^{(1)}$. Los trastornos más frecuentes son las taquicardias, y se clasifican como taquicardias supraventriculares cuando su mecanismo de producción y perpetuación está por encima de la bifurcación del haz de His, y ventriculares cuando está por debajo de este. También se pueden dividir en paroxísticas cuando son de inicio y fin súbitos, y no paroxísticas cuando se inician de manera gradual y terminan de la misma manera ${ }^{(2-4)}$. La incidencia varía desde $0,1 \%$ hasta $0,4 \%$ en la población pediátrica ${ }^{(5-9)}$, pero algunos estudios latinoamericanos aislados muestran una incidencia mucho mayor, llegando hasta $0,86 \%{ }^{(10)}$; lamentablemente no tenemos datos nacionales debido, probablemente, a la carencia de centros especializados en el tratamiento invasivo de trastornos del ritmo cardiaco en niños.

La clínica, muchas veces inespecífica, va desde formas asintomáticas hasta formas severas que debutan con notable repercusión hemodinámica, cuya expresión ecocardiográfica es la disminución de la fracción de eyección que, asociada a dilatación de cavidades cardiacas, es catalogada como taquicardiomiopatía (11). El diagnóstico clínico es complicado en pacientes pequeños, sobre todo los menores de 1 año, ya que los síntomas suelen ser muy inespecíficos, en algunos casos llegándose a confundir con cuadros digestivos como enfermedad por reflujo gastroesofágico, por los vómitos asociados ${ }^{(2,8,12)}$. El diagnóstico estricto se realiza mediante el trazado electrocardiográfico (ECG), es sencillo si se cuenta con documentación delataquicardia;sinembargo, dichadocumentación muchas veces se muestra evasiva, ya que algunos eventos son de corta duración, por tanto, es hacia ella donde van dirigidas nuestras maniobras diagnósticas y, posteriormente, terapéuticas. En niños con corazones estructuralmente normales, el mecanismo más común de taquicardia supraventricular es la reentrada auriculoventricular por vía accesoria, cuya ubicación es variable, seguida por la taquicardia por reentrada intranodal y la taquicardia auricular focal. La primera es más común en niños pequeños, mientras que la taquicardia por reentrada nodal o intranodal tiende a ocurrir en adolescentes y jóvenes ${ }^{(2,13-15)}$. El estudio electrofisiológico, junto con la ablación cardiaca, generalmente por radiofrecuencia, forman parte de nuestro arsenal diagnóstico y terapéutico, y es el único método por el cual determinamos el mecanismo exacto de la taquicardia, pudiéndose identificar la ubicación de la vía accesoria, si es que está presente; sin embargo, cuenta con ciertas restricciones respecto al peso mínimo recomendado (el paciente debe pesar más de 15 $\mathrm{kg})^{(1)}$. Respecto al tratamiento médico, existen muchas alternativas, tanto en terapia única o combinada; lamentablemente, estos medicamentos no están exentos de efectos secundarios, incluso se describen problemas tiroideos, pulmonares e intestinales asociados con uso crónico de amiodarona, por lo cual la posibilidad de un tratamiento curativo resulta bastante atractiva $(1,5,16,17)$. Es importante mencionar que existen escasos reportes que incluyan pacientes pediátricos en nuestro país ${ }^{(18)}$, nuestra institución realizó su primer procedimiento en octubre de 2018, constituyendosé en la actualidad como el principal centro de referencia para ablaciones pediátricas en el Ministerio de Salud.

Por tanto, en el Perú, el diagnóstico y tratamiento oportuno de las taquicardias en pediatría, evitando el impacto en el deterioro de la función cardiaca y en la calidad de vida del paciente es imperativo. Para tal fin, analizamos una cohorte nacional de pacientes pediátricos que fueron sometidos a un estudio electrofisiológico y ablación por radiofrecuencia. El objetivo del estudio fue describir las características clínicas, electrofisiológicas y terapéuticas de la población pediátrica con taquicardia supraventricular, sometida a ablación por radiofrecuencia, en una institución pública del Perú.

\section{Materiales y métodos}

\section{Diseño y población de estudio}

Estudio observacional, descriptivo y retrospectivo, realizado entre octubre de 2018 y septiembre de 2021 en el Instituto Nacional de Salud del Niño San Borja, en Lima, Perú. La población estuvo compuesta por todos los pacientes menores de 18 años con diagnóstico de taquicardia supraventricular, que fueron sometidos a un estudio electrofisiológico con ablación por radiofrecuencia.

\section{Variables de estudio}

Se estudiaron las siguientes variables: edad, sexo, peso, procedencia, fármacos antiarrítmicos, presencia de cardiopatía estructural, síntomas presentes antes de la ablación comorbilidades, taquicardia documentada, y preexcitación en reposo. Respecto al procedimiento, se incluyó el mecanismo de la arritmia; la ubicación y el número de vías accesorias; la duración del procedimiento; la tasa de éxito, las complicaciones, y las reintervenciones. La tasa de éxito y fracaso se definieron al final de la intervención. La tasa de recurrencia se definió como la necesidad de reintervención mediante un segundo estudio electrofisiológico y ablación por radiofrecuencia.

\section{Procedimientos}

Los datos se obtuvieron delas historias clínicasy de la base de datos de cardiología. La vía accesoria fue clasificada según su ubicación anatómica (Figura 1) ${ }^{(14)}$. La aplicación de radiofrecuencia en la zona crítica fue titulada de acuerdo con el criterio del operador principal. Una vez alcanzado el resultado deseado para cada tipo 
de arritmia, se consideró éxito agudo a la ausencia de recurrencia de la arritmia clínica dentro de los 30 min de observación.

\section{Análisis de datos}

Se realizó un análisis descriptivo utilizando medidas de tendencia central, medidas de dispersión, frecuencias absolutas y frecuencias relativas. Los resultados se presentan como números absolutos y frecuencias relativas. El análisis estadístico se realizó empleando el paquete Stata v15.

\section{Aspectos éticos}

Se obtuvo el consentimiento informado de pacientes (cuando fue posible) y de sus apoderados. El presente trabajo no involucró ninguna intervención o modificación intencionada de las variables biológicas, fisiológicas, psicológicas o sociales de los pacientes pediátricos incluidos en el estudio. El estudio fue aprobado por el comité de ética institucional.

\section{Resultados}

Durante el período de estudio, 59 pacientes cumplieron los criterios de inclusión, y se realizaron 62 procedimientos (tres pacientes fueron sometidos a dos procedimientos por recurrencia de la taquicardia). La proporción de pacientes con la primera detección de la taquicardia en el primer año de vida fue del 8,5\% (5/59 pacientes); 1 de estos cinco pacientes fue diagnosticado durante el período fetal. El $56 \%$ de pacientes procedía de la región Lima, y el 61\% fueron pacientes de sexo masculino.

\section{Hallazgos clínicos}

Todos los pacientes por encima de los 3 años refirieron palpitaciones, la documentación de la taquicardia en el momento del diagnóstico estaba disponible en 57 de 62 procedimientos $(91,9 \%)$. Solo dos pacientes presentaron dilatación de cavidades, uno de ellos con disminución de la fracción de eyección, y ambos tenían el diagnóstico de taquicardia permanente reciprocante de la unión. No se documentó taquicardia de complejo ancho debido a aberrancia dependiente de la frecuencia o bloqueo de rama del haz de His. La taquicardia antidrómica solo se documentó en un paciente.

\section{Detalles del procedimiento}

Las características de los pacientes y los detalles de la intervención con catéter se muestran en la Tabla 1. El 23\% de los procedimientos correspondieron a pacientes de menos de $15 \mathrm{~kg} ; 47$ procedimientos (75,8\%) consistieron en estudio electrofisiológico y posterior ablación, mientras que en el resto solo se realizó estudio electrofisiológico. La ablación se realizó con energía de radiofrecuencia en todos los procedimientos
Tabla 1. Características de los pacientes y duración de los procedimientos

\begin{tabular}{lc}
\hline Características & $\mathbf{n}=\mathbf{6 2}$ \\
\hline \multirow{2}{*}{ Edad (años) } & $9,91 \pm 5,3$ \\
& (Rango: 22 días -17 años \\
& 3 meses) \\
Peso $(\mathrm{kg})$ & $39 \pm 22,2$ \\
& (Rango: $2,8-78,7)$ \\
Talla $(\mathrm{cm})$ & $132 \pm 33,5$ \\
Tiempo de & (Rango: $42-176)$ \\
procedimiento $(\mathrm{min})$ & $99 \pm 48$ \\
\hline
\end{tabular}

(punta no irrigada $n=1 / 47(2,1 \%)$, punta irrigada $n=46 / 47$ (97,9\%). La tasa de éxito dependió de la taquicardia, así, la tasa de éxito global de la primera ablación con catéter fue del 90,9 y 95,5\% si nos limitamos a taquicardias dependientes de vía accesoria; en pacientes con anomalía de Ebstein, la tasa de éxito fue del $86 \%$. Respecto a pacientes con flutter atrial, la tasa de éxito fue de $87 \%$ (5/6). En pacientes con taquicardia de reentrada del nodo auriculoventricular o taquicardia intranodal, la tasa de éxito fue $100 \%$. La tasa general de complicaciones fue del 1,6\% (1/62). Dentro de las complicaciones, solo se observó la formación de pseudoaneurisma en la zona de punción $(n=1)$, no se registró bloqueo auriculoventricular de primer grado, segundo grado, tercer grado, taponamiento pericárdico, embolia o perforación cardiaca. La tasa de recurrencia fue de 9,1\% (4/44), se realizó reintervención por taquicardia recurrente en 3/44 pacientes $(6,8 \%)$. Dos de los pacientes con recurrencia tuvieron una taquicardia dependiente de vía accesoria (uno de ellos con taquicardia permanente reciprocante de la unión), otro paciente tuvo el diagnóstico de flutter atrial, y el último de taquicardia atrial.

\section{Mecanismos de taquicardia}

La Tabla 2 resume los sustratos de arritmia en el estudio electrofisiológico. En nuestro estudio, la taquicardia mediada por la vía accesoria fue la más común, representando el $60 \%$ de todas las taquicardias. El segundo tipo más común de taquicardia fue la taquicardia auricular focal, seguida por el flutter atrial. En nueve pacientes fue imposible inducir taquicardia en el momento del estudio electrofisiológico, a pesar de esto, la presencia de una vía accesoria fue identificada en estos pacientes mediante análisis electrofisiológicos exhaustivos en cuatro pacientes, los cuales fueron sometidos a ablación. Dentro del grupo de taquicardia auricular focal, el foco se ubicó en la aurícula derecha en todos los casos. Se detectaron tres pacientes con taquicardia intranodal, uno de ellos presentaba doble sustrato arritmogénico (taquicardia intranodal y taquicardia ventricular fascicular). 
Tabla 2. Tipo de taquicardia

\begin{tabular}{lc}
\hline Tipo de taquicardia & $\mathbf{n = 5 9 ( \% )}$ \\
\hline Mediado por vía accesoria & $36(60)$ \\
- TRAV con preexcitación & $13(21,7)$ \\
- TRAV sin preexcitación & $20(33,3)$ \\
- Taquicardia reciprocante permanente de la unión (Coumel) & $3(5)$ \\
Taquicardia por reentrada intranodal (TIN) & $3(5)$ \\
Taquicardia atrial & $7(11,7)$ \\
Flutter atrial & $6(10)$ \\
No inducible & $5(8,3)$ \\
Otros ** & $3 *(5)$ \\
\hline
\end{tabular}

* 1 paciente tuvo más de 1 tipo de taquicardia.

** Otros = fibrilación auricular $(n=1)$, taquicardia ventricular fascicular $(n=2)$.

TRAV: Taquicardia por reentrada auriculo-ventricular

\section{Cardiopatías congénitas}

En nuestro estudio 20 pacientes presentaban alguna cardiopatía estructural, la más frecuente fue la anomalía de Ebstein en 12 casos (12/59; 20,3\%), seguido por la conexión venosa anómala pulmonar total $(4 / 59 ; 6,8 \%)$ quienes presentaron flutter atrial en el periodo posoperatorio tardío. De los 12 pacientes con anomalía de Ebstein, cinco fueron sometidos solo a estudio electrofisiológico (por no ser susceptible de tratamiento), el resto $(7 / 12)$ fue sometido a estudio electrofisiológico y ablación por radiofrecuencia, seis de ellos presentaban taquicardia por reentrada atrioventricular (cuatro con preexcitación manifiesta y dos con vía oculta) y 1 paciente presentaba taquicardia atrial. Por otro lado, todos los pacientes con conexión venosa anómala pulmonar total presentaron flutter atrial y fueron sometidos a ablación (4/4). Otros diagnósticos fueron estenosis pulmonar $(n=2)$ y tetralogía de Fallot $(n=2)$.

\section{Número de vías accesorias}

Treinta y seis pacientes presentaron una taquicardia mediada por la presencia de alguna vía accesoria. Una sola vía accesoria estuvo presente en 33 de 36 (91,7\%), se observaron dos vías accesorias en 2 de 36 pacientes (5,6\%). Tres vías accesorias estuvieron

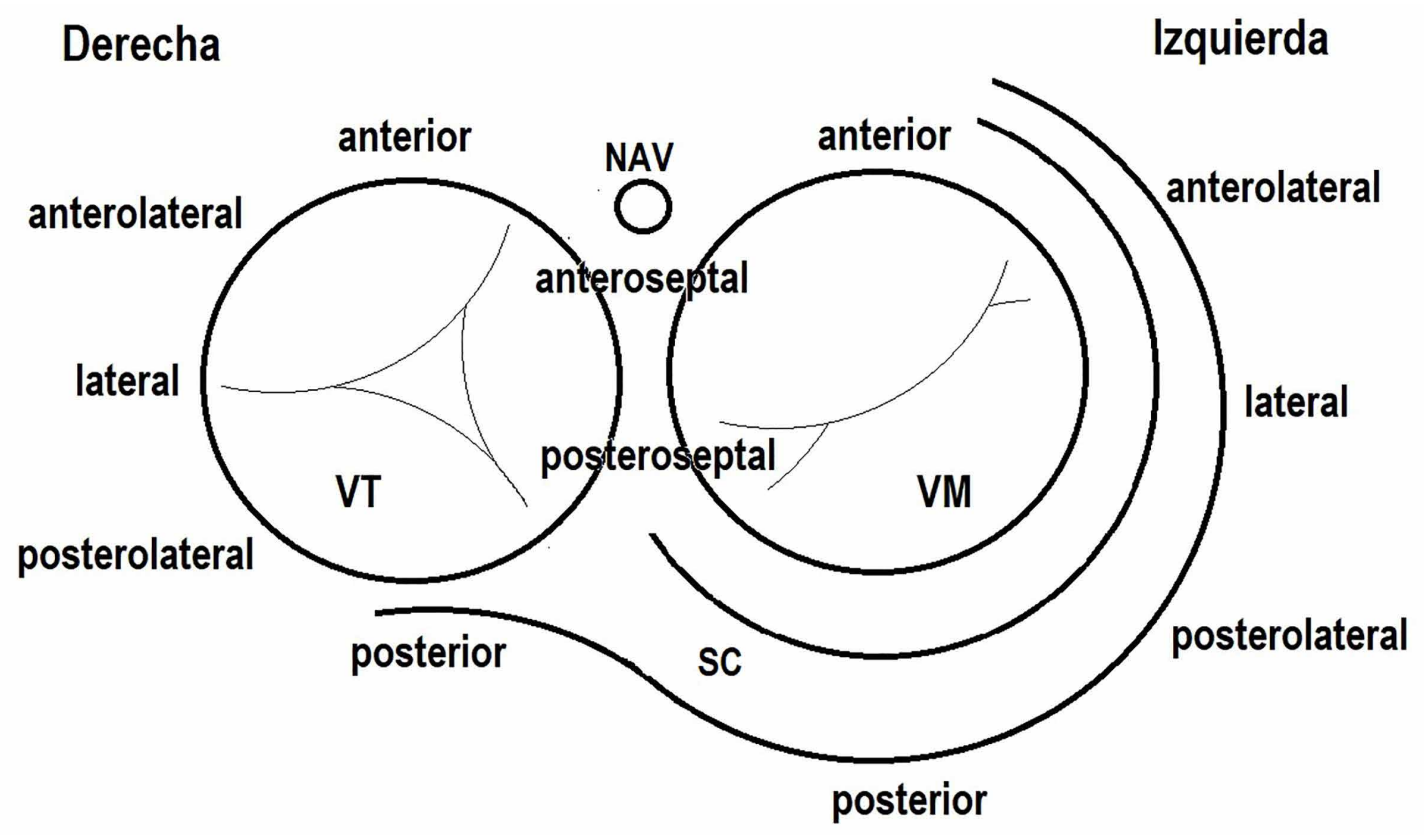

Figura 1. Representación esquemática de la unión atrioventricular en la proyección oblicua anterior izquierda. 
presentes en 1 de 36 pacientes (2,7\%). En pacientes con corazones estructuralmente normales solo se encontró una vía accesoria, los casos con dos y tres vías accesorias corresponden a pacientes con anomalía de Ebstein.

\section{Ubicación de vías accesorias}

La ubicación de las vías accesorias se muestra en las Figuras 2 y 3. Allí se observa una tendencia a favor de las vías del lado izquierdo respecto a las vías del lado derecho (25 versus 5 ) en la población con corazón estructuralmente normal. La ubicación lateral izquierda fue la más común. Dentro del grupo de pacientes con anomalía de Ebstein, todas las vías accesorias corresponden a una ubicación derecha, siendo las más frecuentes la posterior y la posteroseptal.

\section{Terapia con fármacos antiarrítmicos}

En las semanas previas a la ablación con catéter, 21 de 59 (35,6\%) no recibían medicación, en el resto de los pacientes la medicación fue suspendida $72 \mathrm{~h}$ antes del procedimiento. Los agentes bloqueadores de los receptores beta fueron en general los fármacos más utilizados (32/59; 54,2\%), a la cabeza el propranolol con 29 pacientes. No se registró el uso aislado de amiodarona, pero si hubo un caso de asociación de amiodarona y propranolol (Figura 4). En todos los casos se suspendió la medicación antiarrítmica luego de la ablación.

\section{Discusión}

En nuestro estudio encontramos, que se detectó taquicardia desde la etapa fetal, las palpitaciones fue el síntoma más frecuente, la taquicardia se asocio a miocardiopatía en una pequeña proporción de casos, la taquicardia de reentrada auriculoventricular fue la más común, representando el $60 \%$ de todo nuestro registro, la taquicardia se asocio a cardiopatía estructural en $34 \%$ de los casos, siendo la más frecuente la anomalía de Ebstein, la tasa de éxito global de la ablación fue cercana al $90 \%$ sin asociarse a complicaciones mayores, incluso en procedimientos en menores de $15 \mathrm{~kg}$.

La mayor incidencia de vías accesorias en pacientes pediátricos podría explicarse por su patogenia y por cambios posnatales propios del miocardio que acontecen con el aumento de la edad ${ }^{(2-4)}$. En el desarrollo normal del corazón, la progresiva separación anatómica y eléctrica entre las masas musculares de las aurículas y los ventrículos, inicialmente continuas, tienen un rol preponderante, ya que, a la edad gestacional de 20 semanas, solo existe una conexión, el haz de His. Una vía accesoria es el resultado de la falta de regresión de una de estas conexiones musculares y, por lo tanto, a menudo está presente desde nacimiento. Después del parto, la separación puede progresar aun más. Estos fenómenos están bien documentados en la

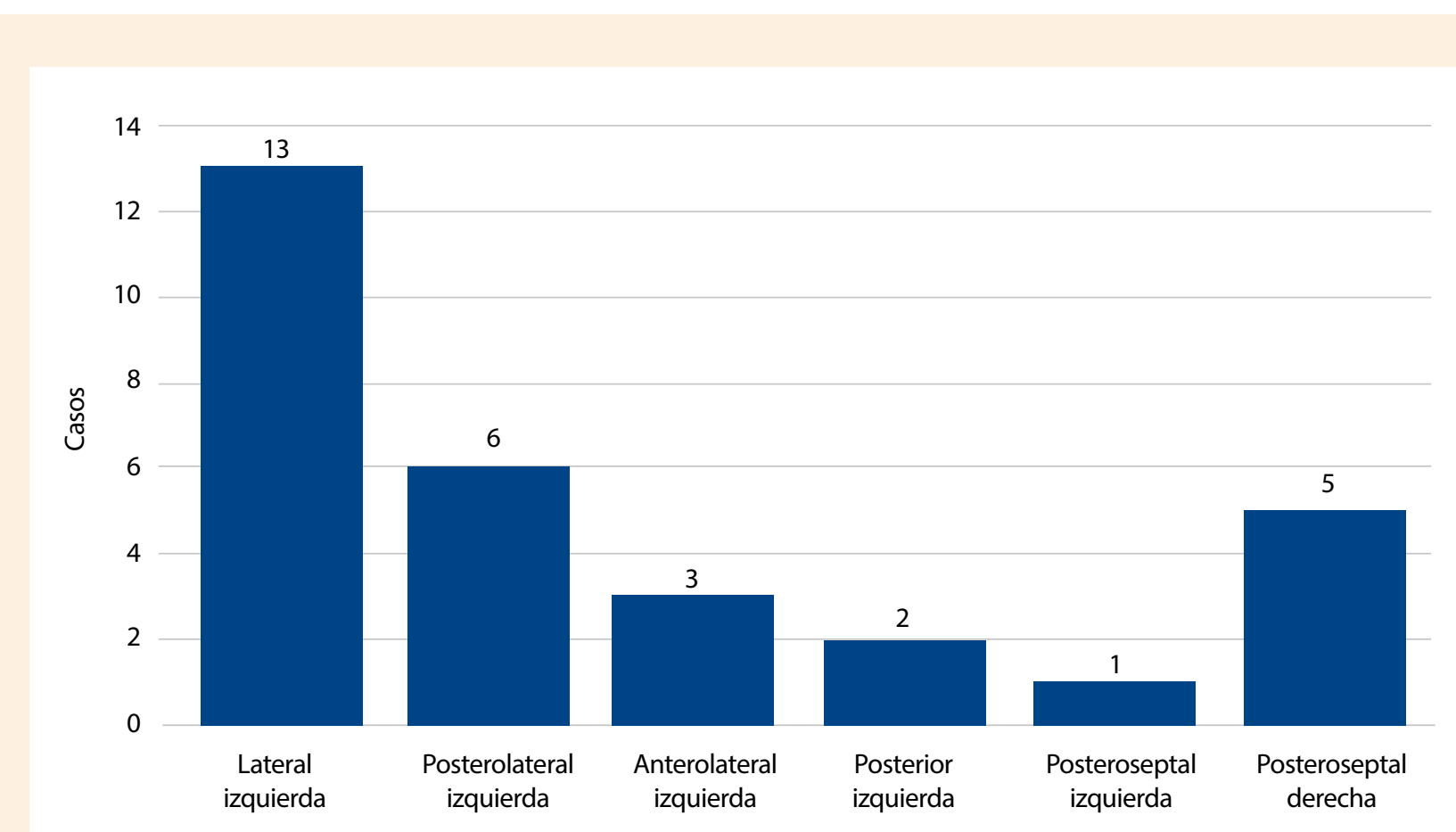

Figura 2. Ubicación de la vía accesoria en corazones estructuralmente normales. 


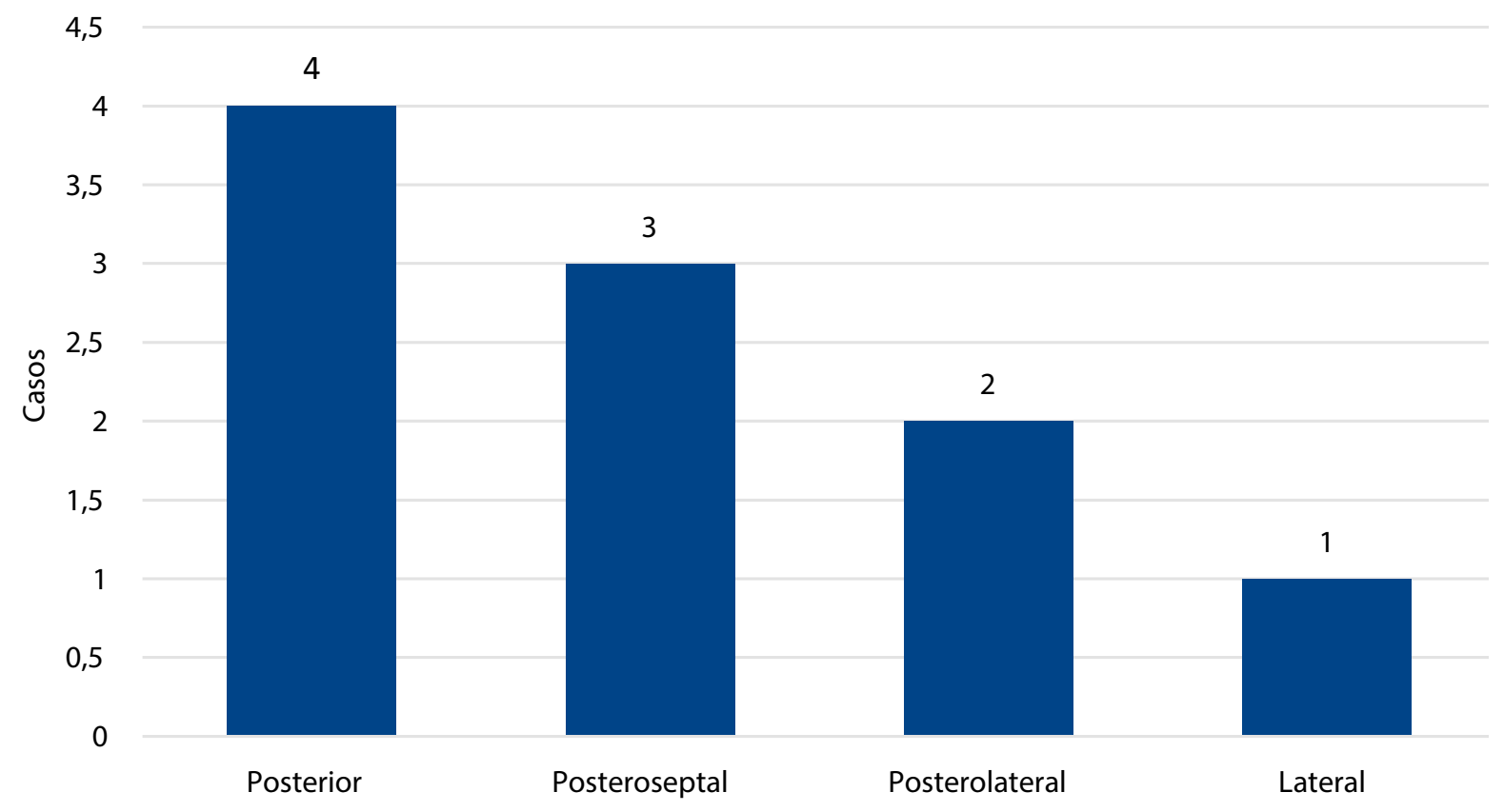

Figura 3. Ubicación de la vía accesoria en anomalía de Ebstein.

preexcitación en pacientes con síndrome de Wolff-ParkinsonWhite. Después de los 5 años es menos probable una regresión espontánea ${ }^{(1,5-9)}$. Otros haces accesorios conducen impulsos solo en dirección retrógrada, estas se conocen como vías ocultas, y el electrocardiograma durante el ritmo sinusal es normal $(3,4,7,25)$. Las vías accesorias del lado izquierdo son más comunes que las vías del lado derecho en pacientes con corazones estructuralmente normales. Esta se aplica a todos los grupos de edad, y también puede evidenciarse en nuestro grupo de estudio. La taquicardia intranodal es más frecuente en adolescentes y adultos jóvenes, en

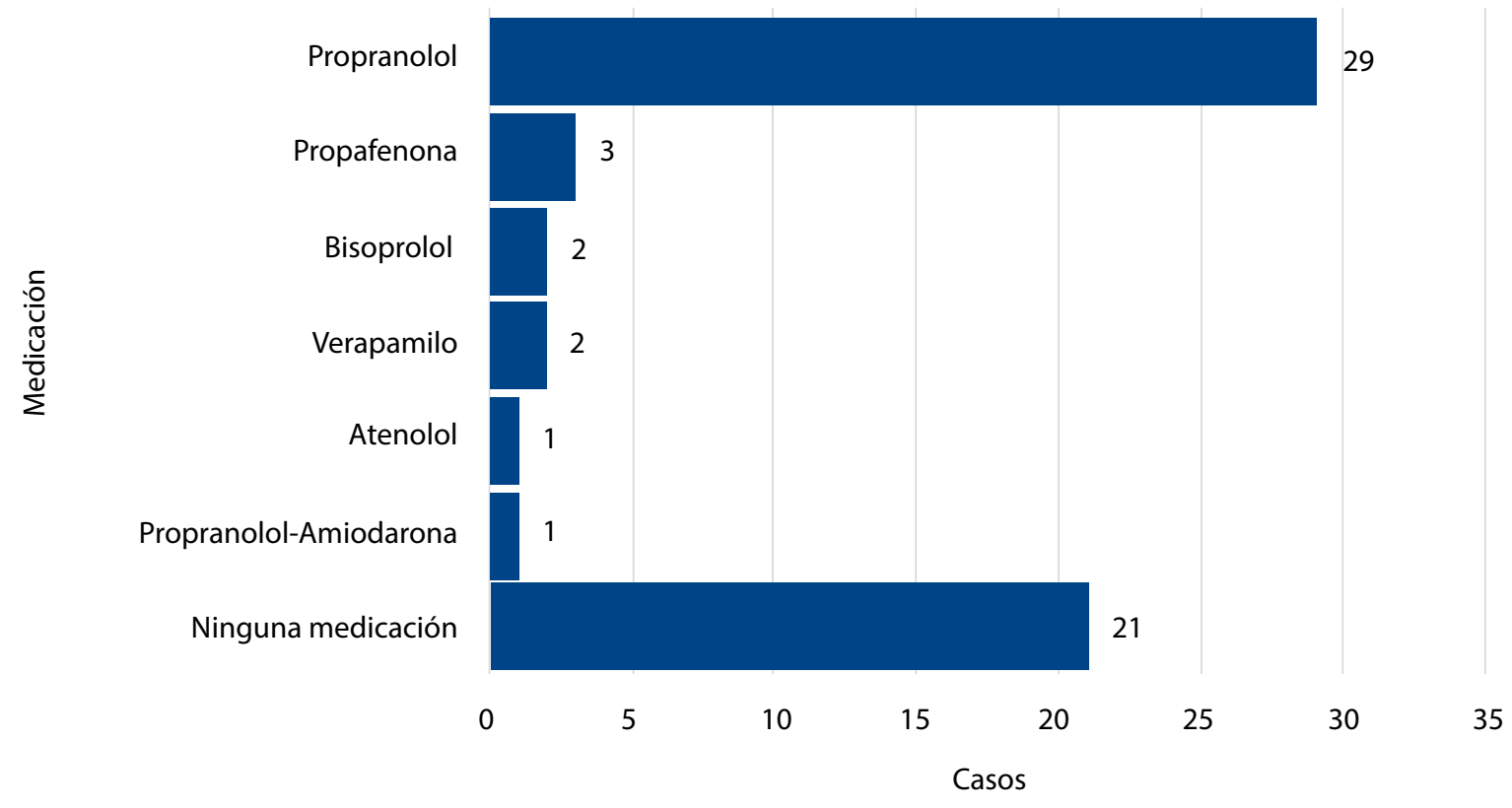

Figura 4. Medicación antiarrítmica usada para el tratamiento de la taquicardia en pediatría. 
nuestra serie solo detectamos tres casos, uno de ellos con doble sutrato arritmogénico, que incluía una taquicardia ventricular fascicular; la taquicardia auricular no se relaciona con la edad, y ocupó una porción importante en nuestro estudio. El mismo resultado ya se muestra en estudios anteriores ${ }^{(2,3,8,13,21,22)}$. Si nos referimos a pacientes con cardiopatías congénitas, en nuestro estudio identificamos a doce pacientes con anomalía de Ebstein, la condición anatómica más relacionada con la presencia de vías accesorias, las cuales pueden ser múltiples, la tasa de éxito referida es mayor a $80 \%{ }^{(3,23,24)}$, en nuestro caso el éxito llegó a $86 \%$.

Una revisión de taquicardia supraventricular en neonatos resalta que el flutter atrial ocupa un lugar importante por detrás de los mecanismos mencionados previamente, la mayoría en corazones estructuralmente normales y con buena respuesta a terapia médica ${ }^{(20)}$, en nuestro estudio todos los casos sometidos a ablación fueron casos posoperatorios. La taquicardia permanente reciprocante de la unión es un subtipo de taquicardia por reentrada atrioventricular, fue descrita por Coumel en 1967, pero fue reconocida mucho antes por Gallavardin en 1927, la principal característica es que se asocia a una vía accesoria con características decrementales ${ }^{(19,26-28)}$. En nuestro estudio identificamos tres pacientes con este diagnóstico, y concordante con la literatura, se trata de pacientes de corta edad, el más joven y pequeño de nuestra serie, que, junto a otro paciente, también lactante menor, presentaron signos compatibles con taquicardiomiopatía. No hubo respuesta a medicación, por lo que de acuerdo con la evidencia la mejor alternativa de tratamiento fue la ablación por radiofrecuencia ${ }^{(19,26)}$.

La presentación clínica abarca un abanico de posibilidades, desde las formas asintomáticas u oligosintomáticas, generalmente palpitaciones, hasta signos de falla cardiaca, otras veces existe sintomatología inespecífica, como vómitos e irritabilidad, especialmente en niños pequeños ${ }^{(2)}$. En nuestra serie la totalidad de pacientes mayores de 3 años manifestaron la sensación de palpitaciones, es probable que en niños menores de esta edad no se pudiera objetivar este tipo de molestia. Se detectaron dos casos con compromiso ecocardiográfico compatible con taquicardiomiopatía, ambos con el diagnóstico de taquicardia permanente reciprocante dela unión, quevolvieron a la normalidad entre 3 a 6 meses luego de la ablación exitosa, concordante con lo que describe la literatura $(2,11,20,29,30)$.

El tratamiento farmacológico era de uso frecuente en pacientes sintomáticos. La digoxina y los betabloqueadores son los de mayor uso, seguidos por la amiodarona, flecainide y propafenona. Una buena porción de pacientes usa una combinación de drogas, así tenemos la digoxina y propranolol, o propafenona y propranolol, o altas dosis de un solo medicamento, generalmente propranolol ${ }^{(1,2,31,32)}$. El uso de estos fármacos de manera crónica no está exento de eventos secundarios, el máximo representante es la amiodarona, que tiene efectos sistémicos indeseados en la tiroides, pulmones, intestinos y piel ${ }^{(1)}$. En este estudio más del $50 \%$ de pacientes usaron betabloqueadores, con el propranolol a la cabeza, solo detectamos un caso de terapia combinada, la cual incluía amiodarona, medicamento que debe ser reservado para casos puntuales con pobre respuesta a otra medicación

En la práctica clínica, el estudio electrofisiológico invasivo y la ablación por radiofrecuencia se pospone en pacientes pequeños, si es posible, hasta que el paciente alcance un peso corporal de aproximadamente $15 \mathrm{~kg}$; sin embargo, diversos estudios y el nuestro, con una tasa de éxito de la ablación cercana al 95\% en taquicardia por reentrada atrioventricular y una baja tasa de complicaciones $(1,6 \%)$, demuestran que la realización de esta técnica invasiva es confiable y segura ${ }^{(1,23,33-35)}$. Es importante resaltar que nuestra institución es la primera a nivel del Ministerio de Salud en realizar este procedimiento en niños de menos de 15 $\mathrm{kg}$. Solo fueron sometidos a ablación pacientes con el diagnóstico de taquicardia por reentrada auriculoventricular por vía accesoria, taquicardia intranodal, flutter atrial, taquicardia ventricular fascicular y un solo paciente con taquicardia atrial (niño con anomalía de Ebstein, muy sintomático), ya que la información en pediatría acerca de esta última aún es limitada.

La inclusión de 59 pacientes en este estudio proporciona una importante cantidad de datos relevantes sobre nuestra realidad nacional.Sin embargo, los hallazgos no se pueden generalizarya que corresponde a una muestra pequeña de un único centro. Por otro lado, es importante resaltar que este estudio, aun siendo descriptivo, abre las puertas para futuras investigaciones en pediatría.

En conclusión, el sustrato de la taquicardia supraventricular más frecuente en pacientes pediátricos fue la presencia de alguna vía accesoria. La taquicardia reentrante mediada por vía accesoria fue el mecanismo más común; las vías accesorias del lado izquierdo fueron la causa más frecuente de taquicardia supraventricular en pacientes con corazón estructuralmente normal, mientras que en pacientes con anomalía de Ebstein la principal ubicación fue la derecha. La ablación con catéter de radiofrecuencia muestra una alta tasa de éxito y una baja tasa de complicaciones, independientemente del peso, resultados satisfactorios, pero se que requieren de mayores estudios para ser generalizados.

\section{Contribuciones de los autores}

LAMQ y FCC participaron en la concepción y diseño del trabajo, recolección/obtención de resultados, análisis e interpretación de datos, redacción del manuscrito, revisión crítica del manuscrito y la aprobación de su versión final 


\section{Referencias bibliográficas}

1. Brugada J, Blom N, Sarquella-Brugada G, Blomstrom-Lundqvist C, Deanfield J, Janousek J, et al. Pharmacological and nonpharmacological therapy for arrhythmias in the pediatric population: EHRA and AEPC-Arrhythmia Working Group joint consensus statement. Europace. 2013;15(9):1337-82. doi: 10.1093/europace/ eut082.

2. Tunca Sahin G, Ozturk E, Kasar T, Guzeltas A, Ergul Y. Sustained tachyarrhythmia in children younger than 1 year of age: Six-year single-center experience. Pediatr Int. 2018;60(2):115-21. doi: 10.1111/ ped.13445.

3. Wren C. Concise Guide to Pediatric Arrhythmias. 1st ed. Oxford: Wiley - Blackwell; 2011.

4. Richardson C, Silver ES. Management of Supraventricular Tachycardia in Infants. Pediatr Drugs. 2017;19(6):539-51. doi: 10.1007/s40272017-0254-0.

5. Balaguer Gargallo M, Jordán García I, Caritg Bosch J, Cambra Lasaosa FJ, Prada Hermogenes F, Palomaque Rico A. Taquicardia paroxística supraventricular en el niño y el lactante. An Pediatría. 2007;67(2):1338. doi: 10.1016/S1695-4033(07)70573-8.

6. Vos P, Pulles-Heintzberger C, Delhaas T. Supraventricular tachycardia: an incidental diagnosis in infants and difficult to prove in children. Acta Paediatr. 2007;92(9):1058-61.

7. Gándara Ricardo JA, Santander Bohórquez D, Mora Pabón G, Amaris Peña Ó. Taquicardias supraventriculares. Estado del arte. Rev Fac Med. 2016;64(1):111-21.

8. Chu PY, Hill KD, Clark RH, Brian Smith P, Hornik CP. Treatment of supraventricular tachycardia in infants: Analysis of a large multicenter database. Early Hum Dev. 2015;91(6):345-50. doi: 10.1016/j. earlhumdev.2015.04.001.

9. Josephson M, Wellens H. Differential Diagnosis of Supraventricular Tachycardia. Cardiol Clin. 1990;8(3):411-42

10. Hermanni M, Marcano E, Sparano A, Machado L, Guerra Y, Akel G, et al. Taquicardia supraventricular en pediatría. Presentación clínica y manejo. Experiencia del servicio de cardiología hospital de Niños JM de los Ríos. Caracas, Venezuela. 2001-2011. Arch Venez Puer Ped. 2011;74(4):143-50.

11. Gozar L, Marginean C, Toganel R, Muntean I. The role of echocardiography in fetal tachyarrhythmia diagnosis. A burden for the pediatric cardiologist and a review of the literature. Med Ultrason. 2017;19(2):232. doi: 10.11152/mu-892.

12. Dorostkar PC, Silka MJ, Morady F, Dick M. Clinical course of persistent junctional reciprocating tachycardia. J Am Coll Cardiol. 1999;33(2):366-75.

13. Gonzales C, Scavée C. Long RP Tachycardia in a Toddler with Dilated Cardiomyopathy: What is the Mechanism? Insights Pediatr Cardiol. 2016;1(1):1-4.

14. Taguchi N, Yoshida N, Inden Y, Yamamoto T, Miyata S, Fujita M, et al. A simple algorithm for localizing accessory pathways in patients with Wolff-Parkinson-White syndrome using only the R/S ratio. Journal of Arrhythmia. 2014;30:439-443.

15. Hill AC, Silka MJ, Wee CP, Bar-Cohen Y. Characteristics of Decremental Accessory Pathways in Children. Circ Arrhythm Electrophysiol [Internet]. Noviembre de 2016 [citado 10 de septiembre de 2021];9(11). Disponible en: https://www.ahajournals.org/ doi/10.1161/CIRCEP.116.004190

16. Losek JD, Endom E, Dietrich A, Stewart G, Zempsky W, Smith K. Adenosine and Pediatric Supraventricular Tachycardia in the Emergency Department: Multicenter Study and Review. Ann Emerg Med. 1999;33(2):185-91.

17. Rohit M, Kasinadhuni G. Management of Arrhythmias in Pediatric Emergency. Indian J Pediatr. 2020;87(4):295-304. doi: 10.1007/ s12098-020-03267-2.
18. Montañez R, More L, Mendoza P. First catheter ablations in the Ministry of Health system of Peru: Report of the initial experience. Int J Cardiol Heart Vasc. 2019;24:100402. doi: 10.1016/j.ijcha.2019.100402.

19. Kang KT, Potts JE, Radbill AE, La Page MJ, Papagiannis J, Garnreiter $J M$, et al. Permanent junctional reciprocating tachycardia in children: A multicenter experience. Heart Rhythm. 2014;11(8):1426-32. doi: 10.1016/j.hrthm.2014.04.033.

20. Srinivasan C, Balaji S. Neonatal supraventricular tachycardia. Indian Pacing Electrophysiol J. 2019;19(6):222-31. doi: 10.1016/j. ipej.2019.09.004

21. Refaat MM, Scheinman M, Badhwar N. Narrow Complex Tachycardia. Card Electrophysiol Clin. 2016;8(1):67-9. doi: 10.1016/j. ccep.2015.10.006.

22. Prabhu MA, Anderson RD, Sparks PB. Supraventricular tachycardia with abrupt onset and termination: What is the mechanism? J Cardiovasc Electrophysiol. 2018;29(11):1584-7. doi: 10.1111/ jce.13672.

23. Aykan HH, Karagoz T, Akin A, Irdem A, Ozer S, Celiker A. Results of radiofrequency ablation in children with tachycardia-induced cardiomyopathy. Anadolu Kardiyol Derg Anatol J Cardiol. 2014;14(7):625-30. doi: 10.5152/akd.2014.4937.

24. Chiu S, Wang J, Lu C, Wu K, Tseng W, Wu M. Electrophysiology Study for Complex Supraventricular Tachycardia in Congenital Heart Disease Patients With Single-Ventricle Physiology. J Am Heart Assoc. 2016;5(11):e004504. doi: 10.1161/JAHA.116.004504.

25. Swiderski J, Lees MH, Nadas AS. The Wolff-Parkinson-white syndrome in infancy and childhood. Heart. 1962;24(5):561-80.

26. Herranz Barbero A, Cesar S, Martinez-Osorio J, Margarit A, Moreno J, Campuzano O, et al. Long-term outcome of neonates and infants with permanent junctional reciprocating tachycardia. When cardiac ablation changes natural history. J Electrocardiol. 2019;56:85-9. doi: 10.1016/j.jelectrocard.2019.05.006.

27. Schleich JM, Vaksmann G, Khanoyan P, Rey C, Dupuis C. [Permanent junctional reciprocating tachycardia in children and adolescents. Efficacy of medical treatment]. Arch Mal CoeurVaiss. 1992;85(5):553-9.

28. Lucet V, Do Ngoc D, Sidi D, Batisse A, Fidelle J, Coumel P. [Medical treatment and long-term development of permanent reciprocal tachycardia in children. Apropos of 10 cases followed for 11 years]. Arch Mal Coeur Vaiss. 1985;78(2):210-216.

29. Reyes W, Varela G, Tortajada G, Cortellezzi Z. Miocardiopatía inducida por arritmias. Rev Urug Cardiol. 2019;34(1):122-9.

30. KylatRl,SamsonRA. Permanent junctional reciprocating tachycardia in infants and Children. J Arrhythmia. 2019;35(3):494-8. doi: 10.1002/joa3.12193.

31. Hornik CP, Chu PY, Li JS, Clark RH, Smith PB, Hill KD. Comparative effectiveness of digoxin and propranolol for supraventricular tachycardia in infants. Pediatr Crit Care Med. 2014;15(9):839-45. doi: 10.1097/PCC.0000000000000229.

32. Barton AL, Moffett BS, Valdes SO, Miyake C, Kim JJ. Efficacy and Safety of High-Dose Propranolol for the Management of Infant Supraventricular Tachyarrhythmias. J Pediatr. 2015;166(1):115-8. doi: 10.1016/j.jpeds.2014.08.067.

33. Smith RT, Gillette PC, Massumi A, McVey P, Garson A. Transcatheter ablative techniques for treatment of the permanent form of junctional reciprocating tachycardia in young patients. J Am Coll Cardiol. 1986;8(2):385-90.

34. Balaji S, Gillette PC, Case CL. Successful radiofrequency ablation of permanent junctional reciprocating tachycardia in an 18-month-old child. Am Heart J. 1994;127(5):1420-1.

35. Gaita F, Haissaguerre M, Giustetto C, Fischer B, Riccardi R, Richiardi $\mathrm{E}$, et al. Catheter ablation of permanent junctional reciprocating tachycardia with radiofrequency current. J Am Coll Cardiol. 1995;25(3):648-54. 